\title{
Nampt: Intracellular and Extracellular Influence on Diabetes and Obesity
}

\author{
Kevin G Culligan* \\ Royal College of Surgeons in Ireland, Bahrain
}

Submission: October 02, 2017; Published: October 17, 2017

*Corresponding author: Kevin G Culligan, Royal College of Surgeons in Ireland, Bahrain, Building 2441Road 2835, Busaiteen 228, Kingdom of Bahrain, Bahrain, Tel: +3531-291-2715; Email: kgculligan@gmail.com

\begin{abstract}
The prevalence of Type 2 Diabetes Mellitus (T2DM) as a result of increased Obesity, or disability is increasing rapidly. However, only a relatively small percentage of obese individuals develop T2DM, suggesting the contribution of additional factors to whole body insulin resistance. Pharmacological intervention with several anti-diabetic agents, such as Thiazolidinediones (TZDs) can cause increased weight gain, but remain the only option for the majority of obese individuals. A subset of bioactive peptides released from white adipose tissue, termed adipokines, forms the molecular link between obesity and T2DM, conveying whole body insulin resistance and desensitization. Once such adipokine, Nampt, is a rate-limiting enzyme in the production of $\mathrm{NAD}^{+}$. Nampt, also known as Visfatin or PBEF, plays key role in regulation of intracellular redox reactions through the controlled production of $\mathrm{NAD}^{+}$. This production of $\mathrm{NAD}^{+}$within adipocytes activates downstream enzymes such as SIRT1. Through activation of SIRT1, whole body insulin resistance is prevented by increased Adiponectin release, as well as release of Nampt extracellularly. SIRT1 also inhibits the activation of PPAR $Y$-dependent insulin-resistance genes. As a result, the Nampt-NAD ${ }^{+}$-SIRT1-PPARY axis therefore may offer a novel target for treatment strategies of T2DM.
\end{abstract}

Keywords: Nampt; Visfatin; PBEF; NAD+; Obesity; Metabolic disorders; SIRT1; PPARY; Adiponectin; NF-kB

\section{Introduction}

Obesity is a medical condition relating to accumulation and storage of excess body fat, predisposing to negative health implications. The fundamental cause of obesity is a simple energy imbalance between calorific intake and calorific expenditure. Sedentary lifestyle and an increase in consumed calories are the main contributors to this increase in weight gain [1]. Such is the tightness of the molecular link between obesity and Type 2 Diabetes Mellitus (T2DM), some researchers utilize the term Diabesity in reference to both [2]. T2DM, the most common form of diabetes, is characterized by

A. A lack of insulin due to dysfunctional pancreatic beta cells.

B. Reduced insulin sensitivity.

C. Insulin resistance [3].

Insulin-resistant individuals demonstrate an impaired ability of insulin to stimulate glucose uptake into skeletal muscle, to suppress gluconeogenesis, and to suppress hydrolysis of triglycerides into fatty acids in adipose tissue.

Adipose tissue has long been considered as a storage depot for triglycerides. However, it has become well established that a secretary role exists for adipose tissue [4]. As well as releasing lipids, cytokines such as Tumor Necrosis Factor- $\alpha$ and interleukins, chemokines such as Monocot Chemo attractant Protein 1 (MCP-1) and coagulation factors such as Plasminogen Activator 1 (PAI-1) and adipokines are also secreted [5]. A subset of chemokines specific to adipose tissue, termed adipokines is also released [6]. Some, such as lepton and adiponectin have been highly characterized and their functions clearly elucidated $[7,8]$.

Specifically within adipose tissue, Nicotinamide Adenine Dinucleotide $\left(\mathrm{NAD}^{+}\right)$and its derivatives act as essential coenzymes in cellular redox reactions in all living organisms [9]. The primary function of the $\mathrm{NAD}^{+}$pathway mediates energy metabolism, reductive biosynthesis and anti-oxidation. During cellular redox reactions, the associated coenzymes Nicotinamide (NAM) and Nicotinamide Mononucleotide (NMN) transfer electrons throughout the pathway. In general, $\mathrm{NAD}^{+}$has been shown to be synthesized by two separate paths; de novo synthesis from tryptophan or through the salvage pathway, being formed by the recycling of the other coenzymes Nicotinamide and NMN $[9,10]$. $\mathrm{NAD}^{+}$has also been shown to modulate the activity of essential regulators of cellular longevity [11]. As well as its role in redox reactions, $\mathrm{NAD}^{+}$is also an important signaling molecule, being 
released from cells such as adiposities into systemic circulation [12]. Figure 1 Cyclic production process of $\mathrm{NAD}^{+}$is regulated by the rate-limiting enzyme Nampt. $\mathrm{NAD}^{+}$can activate SIRT1, which can elevate serum levels of Adiponectin through activation of Fox01. $\mathrm{NAD}^{+}$inhibits the transcription activity of PPARY, preventing the transcription of adipocytic genes involved in insulin resistance. SIRT1 can also deacetylate iNampt, releasing it extracellularly. Once released, eNampt can convert Nicotinamide (NAM) to Nicotinamide Mononucleotide (NMN), and generate $\mathrm{NAD}^{+}$in pancreatic beta cells, modulating insulin secretion. The presence of SIRT1 in vascular endothelial cells can regulate expression of cell adhesion molecules, implicated in the vascular pathogenesis of Type 2 Diabetes Mellitus.

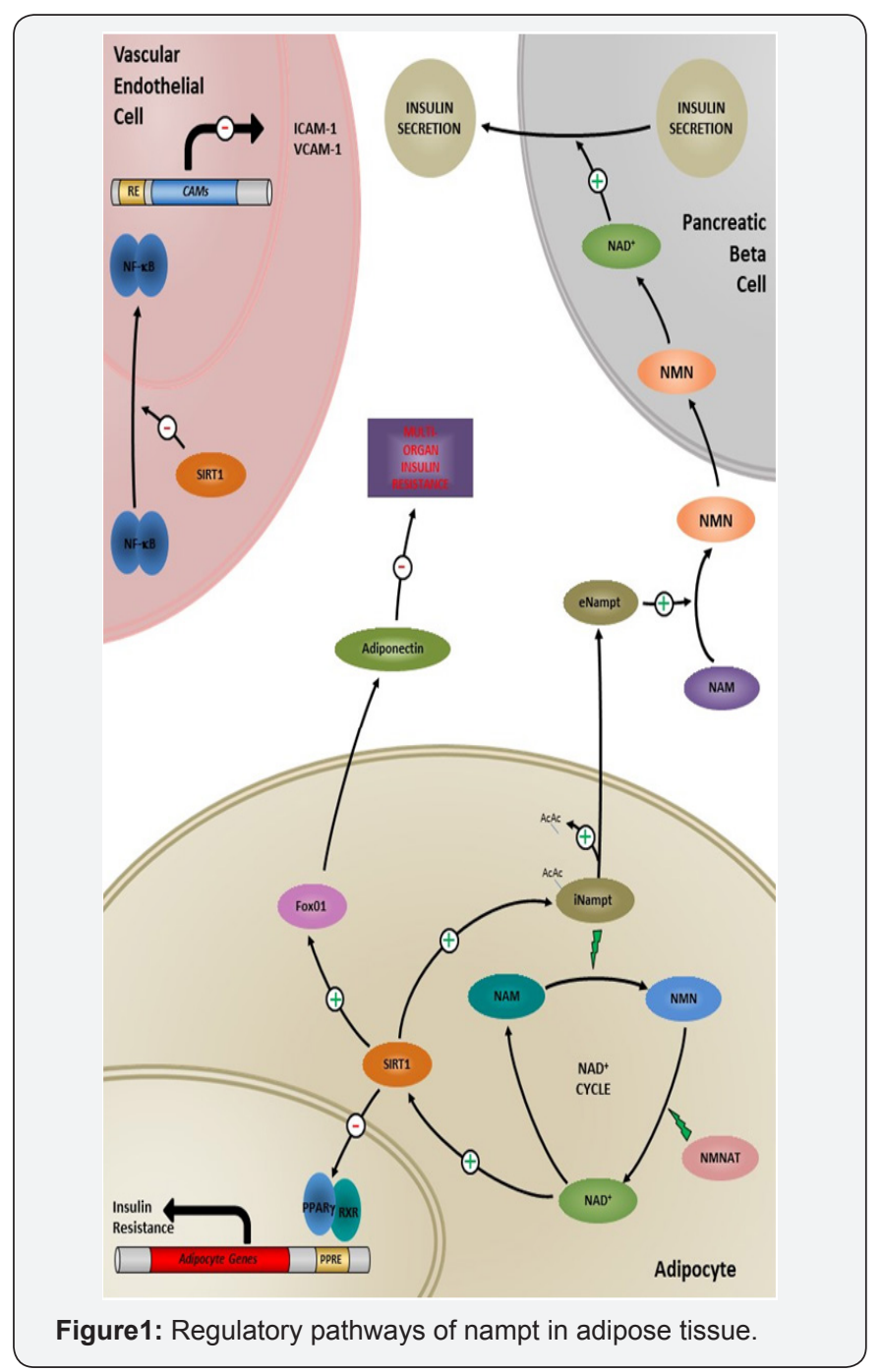

Nampt

Nicotinamide phosphoribosyltransferase (Nampt) is a regulatory enzyme of the $\mathrm{NAD}^{+}$cascade synthesized and released by adiposities, as well as inflammatory cells such as activated macrophages [13]. The enzyme was originally identified as presumptive cytokine termed Pre-B-cell colony-Enhancing Factor (PBEF), due to its isolation and cloning from activated lymphocytes [14]. Visfatin, referring to visceral fat from which the enzyme is derived was separately isolated and shown to possess insulin-mimetic effects, being predominantly secreted from visceral fat [12]. Later, amino acid sequence analysis of Visfatin showed sequence identity to PBEF/Nampt [15].

While all three names (Nampt, PBEF, and Visfatin) can be found used interchangeably in the literature, both the HUGO Gene Nomenclature Committee (HGNC) and the Mouse Genomic Nomenclature Committee (MGNC) have approved the use of Nampt as the official nomenclature of the gene and the gene product. Therefore, Nampt will be used throughout this review.

\section{Genetic Location}

Located on the long arm of chromosome 7(7q22.1-7q31.33) the NAMPT gene is well conserved across species [16]. The gene encodes a $2.4 \mathrm{~kb}$ mRNA sequence, giving rise to a $491 \mathrm{amino}$ acid $52 \mathrm{kDa}$ protein [15]. Interestingly, although Nampt is believed to be secreted, the mature protein sequence lacks both a signal sequence and capsize cleavage site [12]. The mature Nampt protein forms a homodyne, which is essential for catalytic activity of the enzyme. Homodimerization results in the formation of two active sites at the interface of the dimeric protein. It is believed that this region is essential for regulation of the production of NMN from nicotinamide [15].

\section{Types and Regulation}

The mature protein of Nampt exists in two different cellular locations: intracellular Nampt (iNampt) and extracellular Nampt (eNampt) [17]. Although both forms of Nampt are critical ratelimiting enzymes in the production of $\mathrm{NAD}^{+}$, their rate of activity differ, depending on cellular location. In adipose tissue, iNampt is involved in the salvage pathway in the production of $\mathrm{NAD}^{+}$[13]. Within adipose tissue, $\mathrm{NAD}^{+}$is reduced and oxidized continuously. iNampt converts Nicotinamide to NMN, and is the rate limiting enzyme in this step [18]. Subsequently, NMN becomes acetylated by NMNAT, converting it to $\mathrm{NAD}^{+}$. During redox reactions in the cell, $\mathrm{NAD}^{+}$consumers utilize $\mathrm{NAD}^{+}$, converting it to Nicotinamide, which in the salvage cycle becomes available for iNampt to reconvert Nicotinamide to NMN. Depletion of the cellular pool of $\mathrm{NAD}^{+}$is prevented by de novo synthesis of $\mathrm{NAD}^{+}$from tryptophan [10]. The biological role of eNampt has been a matter of much debate. Initially, it was first believed that the systemic release of eNampt was due to dying cells [19]. Later though, it was demonstrated that the secretion of eNampt occurs from adiposities by secretion through a non-classical secretary pathway [20]. The sequence of Nampt lacks both a signal sequence and caspase cleavage site [21]. SIRT1, one such $\mathrm{NAD}^{+}$consumer, deacetylates iNampt, releasing it into the extracellular milieu [22]. Once secreted, eNampt is believed to play several roles essential to glucose homeostasis [20].

It has been demonstrated that eNampt can initiate a dosedependent up regulation of pro- and anti-inflammatory cytokines [23]. With eNampt itself believed to play a role as both a cytokine and adipokine. eNampt mediates the biosynthesis of systemic 
$\mathrm{NAD}^{+}$. This NAD ${ }^{+}$has been shown to be necessary for pancreatic beta cell function, as well as the regulation of Glucose-Stimulated Insulin Secretion (GSIS) [20].

\section{Downstream Mediators}

\section{SIRT1}

SIRT1, a class III his tone deacetylase, belongs to the family of Sit-ins (SIRTs) [24,25]. Through the consumption of NAD+, SIRT1 deactivate downstream proteins on specific lysine residues [26]. Blocking of SIRT1 activity by techniques such as antisense technology can induce adipose tissue infiltration, as well as infiltration of activated macrophages [26]. Conversely, deactivation of Fox01 by SIRT1 in adiposities has been shown to increase levels of Adiponectin, an adipokine conveying sensitivity to insulin. As a result, the pharmacological activation of SIRT1 by agents such as resveratrol is being investigated as a method for increasing insulin sensitivity in diabetic patients [25].

\section{PPARY}

PPARY has long since been a target for investigation for the treatment of obesity-associated insulin resistance, due to its ability to regulate whole-body insulin sensitivity. Interestingly, in adipose tissue SIRT1 suppresses the actions of PPARY, preventing adiposeness $[27,28]$. The actions of SIRT1 on PPARY occur at two distinct sites at a post-translational level. Firstly, deactivation of PPARY suppresses PPARY-dependent insulin-resistance genes [27]. Secondly, through inhibition of phosphorylation of ser273 in PPARY, a subset of PPARY-dependent genes are silenced; these genes controlling glucose metabolism and insulin sensitivity [29]

\section{NF-kB}

A potential role of eNampt in the pathogenesis of vascular inflammation in obesity and T2DM has been suggested [30]. An induction of adhesion molecules such as ICAM-1 and VCAM-1 in response to eNampt has been demonstrated in leukocytes [31], mediated through the induction of the pro-inflammatory transcription factor Nuclear Factor-kB (NF-kB). Induction of NF-kB-mediated matrix metalloproteinase's (MMPs) in vascular endothelial cells was also demonstrated [32]. This suggests a role for eNampt in the development of the vascular complications associated with obesity and T2DM.

\section{Nampt in T2DM}

The development of Nampt knockout mice has supplied evidence of the role of Nampt in both obesity and diabetes. Homozygous deletion of the Nampt gene results in lethality in knockout mice [33]. However, in heterozygote's, impaired glucose tolerance is seen as a result of the regulation of GSIS by Nampt [20]. Loss of Nampt modulates the levels of $\mathrm{NAD}^{+}$in pancreatic beta cells, reducing insulin secretion in response to plasma glucose [33].

In the elderly, T2DM is associated with a progressive decline in pancreatic beta cell function [34]. SIRT1 activation by Nampt- generated $\mathrm{NAD}^{+}$has been implicated in the processes of longevity. Given that SIRT1 is known to positively regulate GSIS, the loss of Nampt through progressive decline in pancreatic beta cell function is believed to contribute to obesity-associated multi-organ insulin resistance [20].

\section{Nampt in Obesity}

In adipose tissue, synthesis of $\mathrm{NAD}^{+}$by Nampt has been shown to be highly sensitive to nutritional changes. These alterations in calorific input have subsequently been implicated in the path physiology of modification of whole-body glucose metabolism and insulin sensitivity [35]. For example, in mice, feeding on hyper calorific diets has been shown to decrease $\mathrm{NAD}^{+}$levels, primarily as a result of a reduction in Nampt [36,37]. Correlation of plasma eNampt levels in human metabolic disorders such as obesity still remains unclear. One of the main reasons for this is a lack of sensitivity of the assay type used to determine serum concentrations [38]. However, a positive correlation between an increase in BMI and Nampt levels has been demonstrated, as well as increased white adipose tissue. Interestingly, gastric banding has been shown to reduce circulating eNampt levels [39]. As assays become more sensitive, more reliable measurement of serum eNampt should give us insight into its effects on metabolic disorders.

\section{Nampt Treatment Strategies}

The use of PPARY agonists has been a mainstay treatment in T2DM in non-obese patients for a considerable time. Activation of PPARY by Thiazolidinediones (TZDs) results in the expression of a number of genes involved in both lipid and glucose metabolism and preadipocyte differentiation. TZDs increase insulin sensitivity, as well as increase utilization of glucose by peripheral tissues [40]. TZDs however have been known to cause hepatic damage, as well as adversely inducing an increase body weight, making them unsuitable for use in obese patients [41].

Since Nampt has been shown to positively regulate PPARY through $\mathrm{NAD}^{+}$-mediated activation of SIRT1, interest has grown in the targeting the Nampt-NAD+-SIRT1-PPARY axis as a novel approach in the treatment of age-related diseases such as ageing and T2DM. A naturally-occurring polyphenolic supplement Resveratrol putatively activates SIRT1 [42]. In animal studies, Resveratrol administration was shown to increase insulin sensitivity and increase longevity of mice on high-calorie diets [43]. More selective and potent SIRT1 activators known as STACs (Synthetic Sit-in-Activating Compounds) such as imidazothiazole are currently under investigation [44].

Given the actions of SIRT1 on glucose homeostasis, an interesting therapeutic approach would be in the development of Nampt-NAD ${ }^{+}$activating compounds. Since activation of SIRT1 is solely dependent on Nampt-NAD+, small molecule activators of these proteins may prove an effective strategy in the treatment of age-related metabolic disorders. 


\section{Current Research in Diabetes \& Obesity Journal}

\section{References}

1. Barnes AS (2011) The epidemic of obesity and diabetes: trends and treatments. Tex Heart Inst J 38(2): 142-144.

2. Al Hannan F, Culligan KG (2015) Human resistin and the RELM of Inflammation in diabesity. Diabetol Metab Syndr 7: 54.

3. Hameed I, Masoodi SR, Mir SA, Nabi M, Ghazanfar K, et al. (2015) Type 2 diabetes mellitus: From a metabolic disorder to an inflammatory condition. World J Diabetes 6(4): 598-612.

4. Tateya S, Kim F, Tamori Y (2013) Recent advances in obesity-induced inflammation and insulin resistance. Front Endocrinol (Lausanne) 4 93.

5. Ronti T, Lupattelli G, Mannarino E (2006) The endocrine function of adipose tissue: an update. Clin Endocrinol (Oxf) 64(4): 355-365.

6. Kos K, Wilding JP (2009) Adipokines: emerging therapeutic targets. Curr Opin Investig Drugs 10(10): 1061-1068.

7. Achari AE, Jain SK (2017) Adiponectin, a Therapeutic Target for Obesity, Diabetes, and Endothelial Dysfunction. Int J Mol Sci 18(6) E1321.

8. Dragano NR, Haddad Tovolli R, Velloso LA (2017) Leptin, neuroinflammation and obesity. Front Horm Res 48: 84-96.

9. Yamaguchi S, Yoshino J (2017) Adipose tissue NAD + biology in obesity and insulin resistance: From mechanism to therapy. Bioessays 39(5).

10. Stromsdorfer KL, Yamaguchi S, Yoon MJ, Moseley AC, Franczyk MP, et al. (2016) NAMPT-Mediated NAD(+) Biosynthesis in Adipocytes Regulates Adipose Tissue Function and Multi-organ Insulin Sensitivity in Mice. Cell Rep 16(7): 1851-1860.

11. Wątroba M, Dudek L, Skoda M, Stangret A, Rzodkiewicz P, et al. (2017) Sirtuins, epigenetics and longevity. Ageing Res Rev 40: 11-19.

12. Fukuhara A, Matsuda M, Nishizawa $M$, Segawa $K$, Tanaka $M$, et al. (2005) Visfatin: a protein secreted by visceral fat that mimics the effects of insulin. Science 307(5708): 426-430.

13. Sonoli SS, Shivprasad S, Prasad CV, Patil AB, Desai PB, et al. (2011) Visfatin-a review. Eur Rev Med Pharmacol Sci 15(1): 9-14.

14. Samal B, Sun Y, Stearns G, Xie C, Suggs S, et al. (1994) Cloning and characterization of the cDNA encoding a novel human pre-B-cell colony-enhancing factor. Mol Cell Biol 14(2): 1431-1437.

15. Wang T, Zhang X, Bheda P, Revollo JR, Imai S, et al. (2006) Structure of Nampt/PBEF/visfatin, a mammalian NAD+ biosynthetic enzyme. Nat Struct Mol Biol 13(7): 661-662.

16. Jia SH, Li Y, Parodo J, Kapus A, Fan L, et al. (2004) Pre-B cell colonyenhancing factor inhibits neutrophil apoptosis in experimental inflammation and clinical sepsis. J Clin Invest 113(9): 1318-2137.

17. Garten A, Schuster S, Penke M, Gorski T, de Giorgis T, et al. (2015) Physiological and pathophysiological roles of NAMPT and NAD metabolism. Nat Rev Endocrinol 11(9): 535-546.

18. Martin PR, Shea RJ, Mulks MH (2001) Identification of a plasmidencoded gene from Haemophilus ducreyi which confers NAD independence. J Bacteriol 183(4): 1168-1174.

19. Stephens JM, Vidal Puig AJ (2006) An update on visfatin/pre-B cell colony-enhancing factor, an ubiquitously expressed, illusive cytokine that is regulated in obesity. Curr Opin Lipidol 17(2): 128-131.

20. Revollo JR, Körner A, Mills KF, Satoh A, Wang T, et al. (2007) Nampt/ PBEF/Visfatin regulates insulin secretion in beta cells as a systemic NAD biosynthetic enzyme. Cell Metab 6(5): 363-375

21. Rongvaux A, Galli M, Denanglaire S, Van Gool F, Drèze PL, et al. (2008) Nicotinamide phosphoribosyl transferase/pre-B cell colony-enhancing factor/visfatin is required for lymphocyte development and cellular resistance to genotoxic stress. J Immunol 181(7): 4685-4695.

22. Yoon MJ, Yoshida M, Johnson S, Takikawa A, Usui I, et al. (2015) SIRT1-Mediated eNAMPT Secretion from Adipose Tissue Regulates Hypothalamic NAD ${ }^{+}$and Function in Mice. Cell Metab 21(5): 706-717.

23. Moschen AR, Kaser A, Enrich B, Mosheimer B, Theurl M, et al (2007) Visfatin, an adipocytokine with proinflammatory and immunomodulating properties. J Immunol 178(3): 1748-1758.

24. Dali Youcef N, Lagouge M, Froelich S, Koehl C, Schoonjans K, et al (2007) Sirtuins: the 'magnificent seven', function, metabolism and longevity. Ann Med 39(5): 335-345.

25. Kitada M, Koya D (2013) SIRT1 in Type 2 Diabetes: Mechanisms and Therapeutic Potential. Diabetes Metab J 37(5): 315-325.

26. Erion DM, Yonemitsu S, Nie Y, Nagai Y, Gillum MP, et al. (2009) SirT1 knockdown in liver decreases basal hepatic glucose production and increases hepatic insulin responsiveness in diabetic rats. Proc Natl Acad Sci USA 106(27): 11288-11293.

27. Qiang L, Wang L, Kon N, Zhao W, Lee S, et al. (2012) Brown remodeling of white adipose tissue by SirT1-dependent deacetylation of Ppary. Cell 150(3): 620-632.

28. Picard F, Kurtev M, Chung N, Topark Ngarm A, Senawong T, et al. (2004) Sirt1 promotes fat mobilization in white adipocytes by repressing PPAR-gamma. Nature 429(6993): 771-776.

29. Choi JH, Banks AS, Kamenecka TM, Busby SA, Chalmers MJ, et al. (2011) Antidiabetic actions of a non-agonist PPAR $\gamma$ ligand blocking Cdk5-mediated phosphorylation. Nature 477(7365): 477-481.

30. Garten A, Petzold S, Schuster S, Körner A, Kratzsch J, (2011) Nampt and its potential role in inflammation and type 2 diabetes. Handb Exp Pharmacol (203): 147-164.

31. Adya R, Tan BK, Punn A, Chen J, Randeva HS (2008) Visfatin induces human endothelial VEGF and MMP-2/9 production via MAPK and PI3K/Akt signalling pathways: novel insights into visfatin-induced angiogenesis. Cardiovasc Res 78(2): 356-365.

32. Adya R, Tan BK, Chen J, Randeva HS (2008) Nuclear factor-kappaB induction by visfatin in human vascular endothelial cells: its role in MMP-2/9 production and activation. Diabetes Care 31(4): 758-760.

33. Zhang LQ, Van Haandel L, Xiong M, Huang P, Heruth DP, et al. (2017) Metabolic and molecular insights into an essential role of nicotinamide phosphoribosyltransferase. Cell Death Dis 8(3): e2705.

34. Basu R, Breda E, Oberg AL, Powell CC, Dalla Man C, et al. (2003) Mechanisms of the age-associated deterioration in glucose tolerance: contribution of alterations in insulin secretion, action, and clearance. Diabetes 52(7): 1738-1748.

35. Chalkiadaki A, Guarente L (2012) Sirtuins mediate mammalian metabolic responses to nutrient availability. Nat Rev Endocrinol 8(5): 287-296.

36. Yoshino J, Mills KF, Yoon MJ, Imai S (2011) Nicotinamide mononucleotide, a key $\mathrm{NAD}^{+}$) intermediate, treats the pathophysiology of diet- and ageinduced diabetes in mice. Cell Metab 14(4): 528-536.

37. Chalkiadaki A, Guarente L (2012) High-fat diet triggers inflammationinduced cleavage of SIRT1 in adipose tissue to promote metabolic dysfunction. Cell Metab 16(2): 180-188.

38. Imai S, Kiess W (2009) Therapeutic potential of SIRT1 and NAMPTmediated NAD biosynthesis in type 2 diabetes. Front Biosci (Landmark Ed) 14: 2983-2995.

39. Haider DG, Schindler K, Schaller G, Prager G, Wolzt M, et al. (2006) Increased plasma visfatin concentrations in morbidly obese subjects 


\section{Current Research in Diabetes \& Obesity Journal}

are reduced after gastric banding. J Clin Endocrinol Metab 91(4): 1578-1581.

40. Thangavel N, Al Bratty M, Javed SA, Ahsan W, Alhazmi HA (2017) Targeting Peroxisome Proliferator-Activated Receptors Using Thiazolidinediones: Strategy for Design of Novel Antidiabetic Drugs. Int J Med Chem 2017: 1069718.

41. Al Salman J, Arjomand H, Kemp DG, Mittal M (2000) Hepatocellular injury in a patient receiving rosiglitazone. A case report. Ann Intern Med 132(2): 121-124.
42. Baur JA, Sinclair DA (2006) Therapeutic potential of resveratrol: the in vivo evidence. Nat Rev Drug Discov 5(6): 493-506.

43. Lagouge M, Argmann C, Hines ZG, Meziane H, Lerin C, et al. (2006) Resveratrol improves mitochondrial function and protects against metabolic disease by activating SIRT1 and PGC-1alpha. Cell 127(6): 1109-1122.

44. Hubbard BP, Sinclair DA (2014) Small molecule SIRT1 activators for the treatment of aging and age-related diseases. Trends Pharmacol Sci 35(3): 146-154

\begin{tabular}{l} 
Your next submission with Juniper Publishers \\
will reach you the below assets \\
- Quality Editorial service \\
- Swift Peer Review \\
- Reprints availability \\
- E-prints Service \\
- Manuscript Podcast for convenient understanding \\
- Global attainment for your research \\
- Manuscript accessibility in different formats \\
( Pdf, E-pub, Full Text, Audio) \\
- Unceasing customer service \\
Track the below URL for one-step submission \\
https://juniperpublishers.com/online-submission.php \\
\hline
\end{tabular}

